
by Rotary Sampling Drills

\author{
Patrick G. Heasler
}

Pacific Northwest National Laboratory

July 1996

Prepared for

Westinghouse Hanford Company and the

U.S. Department of Energy

under Contract DE-AC06-76RLO 1830

Pacific Northwest National Laboratory

Operated by Battelle for the

U.S. Department of Energy 


\section{DISCLAIMER}

This report was prepared as an account of work sponsored by an agency of the United States Government. Neither the United States Government nor any agency thereof, nor Battelle Memorial Institute, nor any of their employees, makes any warranty, express or implied, or assumes any legalliability or responsibility for the accuracy, completeness, or usefulness of any information, apparatus, product, or process disclosed, or represents that its use would not infringe privately owned rights. Reference herein to any specific commercial product, process, or service by trade name, trademark, manufacturer, or otherwise does not necessarily constitute or imply its endorsement, recommendation, or favoring by the United States Government or any agency thereof, or Battelle Memorial Institute. The views and opinions of authors expressed herein do not necessarily state or reflect those of the United States Government or any agency thereof.

\section{PACIFIC NORTHWEST NATIONAL LABORATORY operated by \\ BATTELLE for the \\ UNITED STATES DEPARTMENT OF ENERGY under Contract DE-ACO6-76RLO 1830}

Printed in the United States of America

Available to DOE and DOE contractors from the Office of Scientific and Technical Information, P.O. Box 62, Oak Ridge, TN 37831; prices available from (615) 576-8401.

Available to the public from the National Technical information Service, U.S. Department of Commerce, 5285 Port Royal Rd., Springfield, VA 22161 
PNNL-11222

\title{
Probability of Ignition of Reactive Wastes by Rotary Sampling Drills
}

\author{
P. G. Heasler
}

July 1996

Prepared for

Westinghouse Hanford Company and the

U. S. Department of Energy

under Contract DE-AC06-76RLO 1830

Pacific Northwest National Laboratory

Operated by Battelle for the

U.S. Department of Energy 


\section{Summary}

Sampling with a rotary drill could potentially cause a fire in some Hanford tanks. If the rotary drill experiences a failure while in fuel-rich, dry waste, the waste could be ignited by the hot drill bit. For the saltcake tanks subject to this hazard. this report presents a methodology for calculating the probabilities of fire due to core drill failure. The methodology utilizes sampling data from tank characterization studies to determine the amount of reactive waste in the tanks. The tanks are rated in order of their fire probabilities. and confidence limits are assigned to the estimates. 



\section{CONTENTS}

Summary $\ldots \ldots \ldots \ldots \ldots \ldots \ldots \ldots \ldots \ldots \ldots \ldots \ldots \ldots \ldots$ iii

1 Introduction $\ldots \ldots \ldots \ldots \ldots \ldots \ldots \ldots \ldots \ldots \ldots \ldots \ldots \ldots \ldots$

2 Reactive Waste Calculations $\ldots \ldots \ldots \ldots \ldots \ldots \ldots \ldots \ldots \ldots \ldots$

3 Uncertainty Analysis $\ldots \ldots \ldots \ldots \ldots \ldots \ldots \ldots \ldots \ldots \ldots \ldots$

4 Fire Probabilities $\ldots \ldots \ldots \ldots \ldots \ldots \ldots \ldots \ldots \ldots \ldots \ldots \ldots$

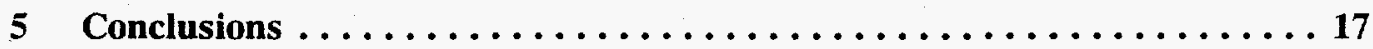

References ................................ 19

\section{LIST OF FIGURES}

Figure 1. Distribution of Calculated Reactive Waste Proportion, Tank BY-104 . . . 7

Figure 2. Proportion of Reactive Waste for Regions 1, 2, and $3 \ldots \ldots \ldots \ldots \ldots$

\section{LIST OF TABLES}

Table 1. Parameters for TOC and Moisture Distributions $\ldots \ldots \ldots \ldots \ldots \ldots$

Table 2. Probability Calculations for Reactive Region $1 \ldots \ldots \ldots \ldots \ldots$

Table 3. Probability Calculations for Reactive Region $2 \ldots \ldots \ldots \ldots \ldots \ldots$

Table 4. Probability Calculations for Reactive Region $3 \ldots \ldots \ldots \ldots \ldots$ 

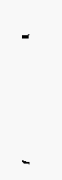


\section{Introduction}

One of the safety concerns regarding the Hanford waste storage tanks is the possibility of a fire in reactive waste caused by rotary sampling drill failure. The object of this report is to estimate the probability of such an occurence during one drill deplovment. In this report. reactive waste is defined as saltcake waste that is dry enough and that contains sufficient total organic carbon (TOC) to burn. The drill failures of concern are those, such as loss of coolant, that would cause ignition of the reactive waste. The probability of a fire occurring due to drill failure is equal to the probability of a drill failure during deplovment times the probability of the drill bit being in reactive waste during failure. In other words.

$$
\begin{aligned}
\operatorname{Pr}(\text { Fire }) & =\operatorname{Pr}(\text { Drill Failure in Reactive Waste }) \\
& =\operatorname{Pr}(\text { Drill Failure }) \cdot \operatorname{Pr}(\text { Drill is in Reactive Waste })
\end{aligned}
$$

This formula assumes that drill failure is no more likely to occur in reactive waste as compared to other waste.

The first term on the right in the equation. the probability that the rotary drill will fail during deployment, has been set to the nominal value of $10^{-4}$, to illustrate the use of the above formula. This nominal value is similar to failure probabilities obtained by the Los Alamos National Laboratory (LANL) for this type of accident.

Estimation of the second term in this equation is the basic objective of this report. To evaluate the second term in the equation, the proportion of reactive waste in each tank is estimated. The proportion of reactive waste in each tank is considered to be equivalent to the chances of a drill failure occuring in reactive waste. This assumption would be true if the sampling location was randomly chosen with respect to the reactive waste. The assumption could also be justified if the reactive waste occured in lavers and drill failure was random in time. The proportion of reactive waste in each tank can be calculated from the results of an earlier ANOVA study [3], which produced distributions of moisture content and TOC for each saltcake tank. 
2 


\section{Reactive Waste Calculations}

These calculations employ three definitions of reactive waste, so that the sensitivity of the desired value (proportion of reactive waste) can be determined. The definitions each equate reactive waste with a certain region in $\left(\mathrm{H}_{2} \mathrm{O}\right.$, TOC $)$ space, to fit the ANOVA data. The three regions are defined as follows:

Region 1: $\mathrm{H}_{2} \mathrm{O}<20 \%$ and $\mathrm{TOC} \%>4.5 \%+0.17 \mathrm{H}_{2} \mathrm{O} \%$

Region 2: $\mathrm{H}_{2} \mathrm{O}<40 \%$ and $\mathrm{TOC} \%>3.0 \%+0.17 \mathrm{H}_{2} \mathrm{O} \%$

Region 3: $\mathrm{H}_{2} \mathrm{O}<20 \%$ and $\mathrm{TOC} \%>3.0 \%+0.17 \mathrm{H}_{2} \mathrm{O} \%$

The proportion of reactive waste in any tank is calculated by integrating the distribution of $\left(\mathrm{H}_{2} \mathrm{O}, \mathrm{TOC}\right)$ in the tank over the reactive region:

$$
\text { Proportion Reactive Waste }=\int_{R_{i}} f(x, y) d x d y
$$

where $x$ and $y$ are the concentrations of $\mathrm{H}_{2} \mathrm{O}$ and $T O C$ respectively, and $R$ represents one of the reactive waste regions defined above. This report gives reactive waste estimates using each of the three reactive regions.

The ANOVA calculations in Reference [3] show that both moisture content and TOC are $\log$-normally distributed (approximately). Thus, the form of the distribution function $f(x, y)$ is

$$
f(x, y)=C_{0} \exp \left(-\frac{1}{2}\left[\begin{array}{c}
\log (x)-\mu_{1} \\
\log (y)-\mu_{2}
\end{array}\right]^{T}\left[\begin{array}{cc}
\sigma_{1}^{2} & \rho \sigma_{1} \sigma_{2} \\
\rho \sigma_{1} \sigma_{2} & \sigma_{2}^{2}
\end{array}\right]^{-1}\left[\begin{array}{l}
\log (x)-\mu_{1} \\
\log (y)-\mu_{2}
\end{array}\right]\right)
$$

where subscripts 1 and 2 refer to moisture content and TOC respectively, $\mu$ is the estimated mean of the $\log$ values for the tank, $\sigma^{2}$ is their estimated variability, $\rho$ is the correlation factor between moisture content and TOC, and the constant $C_{0}$ in the formula is defined as:

$$
C_{0}=\frac{1}{2 \pi \sqrt{1-\rho^{2}} \sigma_{1} \sigma_{2}}
$$

It should also be noted that the log's in the above equation are base 10 logarithms.

The proportion of reactive waste in a tank (and thus, the probability of drill failure in the reactive waste) can be calculated from Equations 1 and 2, given suitable estimates of the distribution parameters $\mu_{1}, \mu_{2}, \sigma_{1}, \sigma_{2}$, and $\rho$. These parameter estimates were obtained for each saltcake tank from References [3] and [2], and are listed in Table 1, along with the standard error (StdErr) and degrees of freedom (DOF) for each estimate. The other parameter needed to solve Equations 1 and 2 is the correlation factor $\rho$. This parameter was assumed equal to 0.39 , based on [3]. 
It should be noted that the integral and distribution Equations 1 and 2 apply only to the saltcake phase of waste in the tank. When applied to the data from Reference [2]. these equations yield the proportion of reactive waste in the saltcake. To produce the proportion of reactive waste to the entire waste content, one must multiply by a factor representing the proportion of saltcake in the tank. These factors have been estimated from [5].

Table 1 contains the parameters that define the TOC and moisture distributions for each saltcake tank. The estimates in this table are in units of $\log _{10}$ of weight percent. The standard error columns in the table represent the uncertainty associated with the estimate. and the "DOF" represent the amount of data used to estimate the sigma parameters. The DOF determines the uncertainty in the sigma s. 
Table 1: Parameters for TOC and Moisture Distributions

\begin{tabular}{|c|c|c|c|c|c|c|c|c|}
\hline \multirow[b]{3}{*}{ Tank } & \multicolumn{4}{|c|}{ TOC Parameters } & \multicolumn{4}{|c|}{$\mathrm{H}_{2} \mathrm{O}$ Parameters } \\
\hline & & $\mu_{2}-$ & & $2-$ & & $\mu_{1}-$ & & $\sigma_{1}$ \\
\hline & Est & StdErr & Est & DOF & Est & StdErr & Est & DOF \\
\hline A101 & -0.23 & 0.18 & 0.49 & 8 & 1.59 & 0.05 & 0.08 & 8 \\
\hline A 102 & -0.23 & 0.18 & 0.49 & 8 & 1.63 & 0.11 & 0.08 & 8 \\
\hline AX101 & -0.23 & 0.18 & 0.49 & 8 & 1.63 & 0.11 & 0.08 & 8 \\
\hline AX102 & -0.25 & 0.17 & 0.49 & 8 & 1.63 & 0.11 & 0.08 & 8 \\
\hline AX103 & -0.25 & 0.17 & 0.49 & 8 & 1.63 & 0.11 & 0.08 & 8 \\
\hline B102 & -0.25 & 0.17 & 0.49 & 8 & 1.63 & 0.11 & 0.08 & 8 \\
\hline B104 & -0.25 & 0.17 & 0.49 & 8 & 1.63 & 0.11 & 0.08 & 8 \\
\hline B105 & -0.29 & 0.20 & 0.49 & 8 & 1.63 & 0.11 & 0.08 & 8 \\
\hline BX105 & -0.25 & 0.17 & 0.49 & 8 & 1.63 & 0.11 & 0.08 & 8 \\
\hline BX110 & -0.25 & 0.17 & 0.49 & 8 & 1.68 & 0.06 & 0.08 & 8 \\
\hline BX111 & -0.25 & 0.17 & 0.49 & 8 & 1.63 & 0.11 & 0.08 & 8 \\
\hline BY101 & -0.19 & 0.16 & 0.49 & 8 & 1.23 & 0.14 & 0.08 & 8 \\
\hline BY 102 & -0.25 & 0.17 & 0.49 & 8 & 1.63 & 0.11 & 0.08 & 8 \\
\hline BY 103 & -0.19 & 0.16 & 0.49 & 8 & 1.63 & 0.11 & 0.08 & 8 \\
\hline BY104 & -0.19 & 0.16 & 0.49 & 8 & 1.21 & 0.04 & 0.08 & 8 \\
\hline BY105 & -0.19 & 0.16 & 0.49 & 8 & 1.63 & 0.11 & 0.08 & 8 \\
\hline BY106 & -0.19 & 0.16 & 0.49 & 8 & 1.63 & 0.11 & 0.08 & 8 \\
\hline BY107 & -0.19 & 0.16 & 0.49 & 8 & 1.23 & 0.14 & 0.08 & 8 \\
\hline BY108 & -0.19 & 0.16 & 0.49 & 8 & 1.23 & 0.14 & 0.08 & 8 \\
\hline BY109 & -0.25 & 0.17 & 0.49 & 8 & 1.63 & 0.11 & 0.08 & 8 \\
\hline BY110 & -0.19 & 0.16 & 0.49 & 8 & 1.23 & 0.14 & 0.08 & 8 \\
\hline BY111 & -0.19 & 0.16 & 0.49 & 8 & 1.23 & 0.14 & 0.08 & 8 \\
\hline BY112 & -0.19 & 0.16 & 0.49 & 8 & 1.23 & 0.14 & 0.08 & 8 \\
\hline S101 & -0.37 & 0.14 & 0.49 & 8 & 1.33 & 0.12 & 0.08 & 8 \\
\hline S102 & -0.37 & 0.14 & 0.49 & 8 & 1.33 & 0.12 & 0.08 & 8 \\
\hline S103 & -0.37 & 0.14 & 0.49 & 8 & 1.33 & 0.12 & 0.08 & 8 \\
\hline S105 & -0.37 & 0.14 & 0.49 & 8 & 0.93 & 0.16 & 0.08 & 8 \\
\hline S106 & -0.37 & 0.14 & 0.49 & 8 & 1.33 & 0.12 & 0.08 & 8 \\
\hline S107 & $-0.3 \bar{T}$ & 0.14 & 0.49 & 8 & 1.33 & 0.12 & 0.08 & 8 \\
\hline $\mathrm{S} 108$ & -0.37 & 0.14 & 0.49 & 8 & 1.33 & 0.12 & 0.08 & 8 \\
\hline S109 & -0.37 & 0.14 & 0.49 & 8 & 1.33 & 0.12 & 0.08 & 8 \\
\hline S110 & -0.37 & 0.14 & 0.49 & 8 & 1.33 & 0.12 & 0.08 & 8 \\
\hline S111 & -0.37 & 0.14 & 0.49 & 8 & 1.20 & 0.04 & 0.08 & 8 \\
\hline $\mathrm{S} 112$ & -0.37 & 0.14 & 0.49 & 8 & 1.33 & 0.12 & 0.08 & 8 \\
\hline SX101 & -0.37 & 0.14 & 0.49 & 8 & 1.09 & 0.11 & 0.08 & 8 \\
\hline SX102 & -0.37 & 0.14 & 0.49 & 8 & 1.12 & 0.06 & 0.08 & 8 \\
\hline$S X$ & -0.37 & .17 & 0.49 & . & 1.09 & 0.1 & 0.08 & 8 \\
\hline SX104 & -0.37 & 0.14 & 0.49 & 8 & 1.07 & 0.06 & 0.08 & 8 \\
\hline
\end{tabular}


Table 1: Parameters for TOC and Moisture Distributions

\begin{tabular}{|c|cccc|cccc|}
\hline & \multicolumn{3}{|c|}{ TOC Parameters } & \multicolumn{3}{c|}{$\mathrm{H}_{2} \mathrm{O}$ Parameters } \\
Tank & \multicolumn{2}{|c}{$-\mu_{2}-$} & $-\sigma_{2} .-$ & \multicolumn{2}{c|}{$-\mu_{1}-$} & $-\sigma_{1}-$ \\
\hline SX105 & -0.37 & StdErr & Est & DOF & Est & StdErr & Est & DOF \\
SX106 & -0.37 & 0.14 & 0.49 & 8 & 1.11 & 0.06 & 0.08 & 8 \\
TX102 & -0.37 & 0.14 & 0.49 & 8 & 1.09 & 0.11 & 0.08 & 8 \\
TX104 & -0.37 & 0.14 & 0.49 & 8 & 1.33 & 0.16 & 0.08 & 8 \\
TX105 & -0.37 & 0.14 & 0.49 & 8 & 0.93 & 0.12 & 0.08 & 8 \\
TX106 & -0.37 & 0.14 & 0.49 & 8 & 0.93 & 0.16 & 0.08 & 8 \\
TX107 & -0.37 & 0.14 & 0.49 & 8 & 1.33 & 0.12 & 0.08 & 8 \\
TX108 & -0.22 & 0.20 & 0.49 & 8 & 1.23 & 0.14 & 0.08 & 8 \\
TX109 & -0.29 & 0.20 & 0.49 & 8 & 1.23 & 0.14 & 0.08 & 8 \\
TX110 & -0.29 & 0.20 & 0.49 & 8 & 1.23 & 0.14 & 0.08 & 8 \\
TX111 & -0.29 & 0.20 & 0.49 & 8 & 1.23 & 0.14 & 0.08 & 8 \\
TX112 & -0.29 & 0.20 & 0.49 & 8 & 1.23 & 0.14 & 0.08 & 8 \\
TX113 & -0.29 & 0.20 & 0.49 & 8 & 1.23 & 0.14 & 0.08 & 8 \\
TX114 & -0.29 & 0.20 & 0.49 & 8 & 1.23 & 0.14 & 0.08 & 8 \\
TX115 & -0.19 & 0.17 & 0.49 & 8 & 1.23 & 0.14 & 0.08 & 8 \\
TX116 & -0.29 & 0.20 & 0.49 & 8 & 1.23 & 0.14 & 0.08 & 8 \\
TX117 & -0.29 & 0.20 & 0.49 & 8 & 1.23 & 0.14 & 0.08 & 8 \\
TX118 & -0.22 & 0.20 & 0.49 & 8 & 1.23 & 0.14 & 0.08 & 8 \\
TY102 & -0.29 & 0.20 & 0.49 & 8 & 1.71 & 0.06 & 0.08 & 8 \\
U102 & -0.19 & 0.17 & 0.49 & 8 & 1.63 & 0.11 & 0.08 & 8 \\
U103 & -0.19 & 0.17 & 0.49 & 8 & 1.63 & 0.11 & 0.08 & 8 \\
U105 & -0.18 & 0.20 & 0.49 & 8 & 1.32 & 0.06 & 0.08 & 8 \\
U106 & -0.19 & 0.17 & 0.49 & 8 & 1.63 & 0.11 & 0.08 & 8 \\
U107 & -0.18 & 0.20 & 0.49 & 8 & 1.33 & 0.12 & 0.08 & 8 \\
U108 & -0.18 & 0.20 & 0.49 & 8 & 1.33 & 0.12 & 0.08 & 8 \\
U109 & -0.18 & 0.20 & 0.49 & 8 & 1.48 & 0.06 & 0.08 & 8 \\
U111 & -0.19 & 0.17 & 0.49 & 8 & 1.58 & 0.05 & 0.08 & 8 \\
\hline
\end{tabular}




\section{Uncertainty Analysis}

No estimate is ever completely accurate. An advantage of ANOVA is that uncertainties (in the form of standard errors) are given for the estimates. Since some estimates contain substantial uncertainty, it is important to account for this in the reactive waste estimates. Simply plugging the ANOVA estimates into formulas 1 and 2 may produce a non-conservative result. To assess the uncertainty, a Monte Carlo simulation [1] was performed.

The Monte Carlo method uses standard Bavesian posterior distributions to describe parameter uncertainty. The log means $\mu$ were assumed to be normally distributed about their estimated means, and the sigma terms $\sigma$.were assumed to have an inverse chi-square distribution defined by their estimates. These distribution parameters were simulated 1000 times, based on their calculated variabilities, and the proportion of reactive waste was calculated for each simulation. For each tank, the results were formed into an empirical distribution as illustrated in Figure 3 for Tank BY-104.

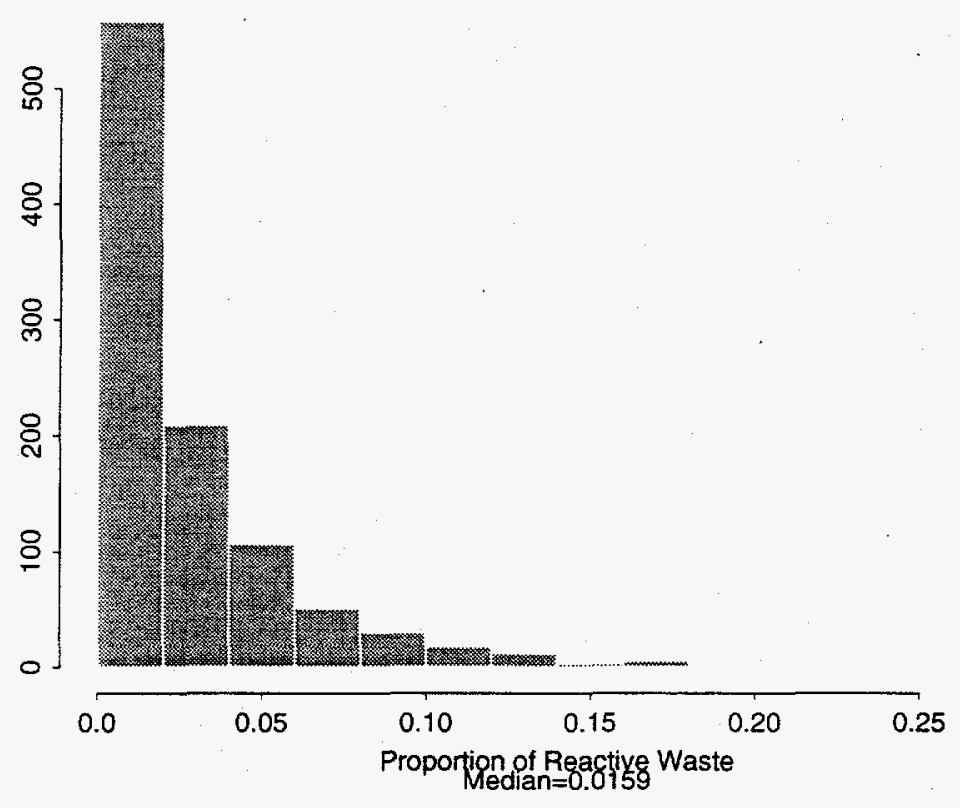

Figure 1: Distribution of Calculated Reactive Waste Proportion, Tank BY-104

This distribution describes the uncertainty associated with the reactive waste calculation for this particular tank. From the figure, the proportion of reactive waste is most probably less than 2 percent. On the other hand. there is a small chance that the proportion might be as high as 15 percent or above. The uncertainty presented in this plot is typical of the reactive waste estimates.

From the Monte Carlo simulations, uncertainties associated with the final results can be 
calculated. In the next section, the final calculated probabilities are reported both as median values (best estimates) and at the upper $95 \%$ confidence level. 


\section{Fire Probabilities}

The following tables present, for each saltcake tank. the calculated estimates of

- the probability of drill failure in reactive waste, and

- the probability of fire due to drill failure.

For all probabilities, both the median values and the upper $95 \%$ confidence level values are tabulated. Tables 2 through 4 show the results for reactive regions 1,2 , and 3, respectively. In each table, the tanks are listed in ascending order of the fire probability. As noted earlier, the fire probabilities in the last two columns are calculated by multiplying the values in the two middle columns by the probability of drill failure, taken as $10^{-4}$ (one chance in 10,000 ). Table 2 also contains the waste volume of the tank, while Table 3 contains the number of TOC observations (samples) made on each tank.

For Region 1, the "best" tank (BX-110) has a zero probability of fire, while the "worst" tank (BY-104) has a probability of about $10^{-6}$ (one chance in a million), indicating that most tanks are safe. For Region 2 . the best and worst probabilities are about $3 \times 10^{-9}$ and $6 \times 10^{-6}$, respectively. As one might expect, this definition yields much higher proportions of reactive waste (and higher probabilities of fire), due principally to the higher moisture content threshold. For Region 3, the results in Table 4 show a range of fire probabilities from zero to $3 \times 10^{-6}$. 
Table 2: Probability Calculations for Reactive Region 1

\begin{tabular}{|c|c|c|c|c|c|c|}
\hline \multirow[b]{2}{*}{ Tank } & \multirow{2}{*}{$\begin{array}{l}\text { Vol } \\
M^{3}\end{array}$} & \multirow{2}{*}{$\begin{array}{l}\text { Fail } \\
\text { Prob }\end{array}$} & \multicolumn{2}{|c|}{ React. Waste } & \multicolumn{2}{|c|}{ Prob Fire } \\
\hline & & & Median & Upper-95\% & Median & Upper- $95 \%$ \\
\hline $\mathrm{BX} 110$ & 785 & $1 \mathrm{e}-04$ & $0.00 \mathrm{e}+00$ & $6.10 \mathrm{e}-08$ & $0.00 \mathrm{e}+00$ & $6.10 \mathrm{e}-12$ \\
\hline TY102 & 243 & $1 e-04$ & $0.00 \mathrm{e}+00$ & $4.41 \mathrm{e}-07$ & $0.00 \mathrm{e}+00$ & $4.41 \mathrm{e}-11$ \\
\hline BX105. & 193 & $1 e-04$ & $4.33 \mathrm{e}-11$ & $5.60 \mathrm{e}-06$ & $4.33 \mathrm{e}-15$ & $5.60 \mathrm{e}-10$ \\
\hline B104 & 1410 & $1 e-04$ & $7.20 \mathrm{e}-11$ & $1.74 \mathrm{e}-05$ & $7.20 \mathrm{e}-15$ & $1.74 \mathrm{e}-09$ \\
\hline BX111 & 800 & $1 e-04$ & $1.59 \mathrm{e}-10$ & $3.82 \mathrm{e}-05$ & $1.59 \mathrm{e}-14$ & $3.82 \mathrm{e}-09$ \\
\hline $\mathrm{B} 102$ & 121 & $1 \mathrm{e}-04$ & $1.67 \mathrm{e}-10$ & $2.82 \mathrm{e}-0.5$ & $1.67 \mathrm{e}-14$ & $2.82 \mathrm{e}-09$ \\
\hline $\mathrm{A} 102$ & 155 & $1 e-04$ & $2.52 \mathrm{e}-10$ & $8.18 \mathrm{e}-05$ & $2.52 \mathrm{e}-14$ & $8.18 e-09$ \\
\hline B105 & 1160 & $1 \mathrm{e}-04$ & $2.82 \mathrm{e}-10$ & $5.01 \mathrm{e}-0.5$ & $2.82 \mathrm{e}-14$ & $5.01 \mathrm{e}-09$ \\
\hline $\mathrm{AX} 102$ & 148 & $1 \mathrm{e}-04$ & $3.63 \mathrm{e}-10$ & $5.29 \mathrm{e}-0.5$ & $3.63 \mathrm{e}-14$ & $5.29 \mathrm{e}-09$ \\
\hline U103 & 1770 & $1 e-04$ & $4.54 \mathrm{e}-10$ & $7.20 \mathrm{e}-05$ & $4.54 \mathrm{e}-14$ & 7.20e-09 \\
\hline AX101 & 2830 & $1 e-04$ & $5.61 \mathrm{e}-10$ & $6.54 \mathrm{e}-05$ & $5.61 \mathrm{e}-14$ & $6.54 \mathrm{e}-09$ \\
\hline BY 103 & 1520 & $1 \mathrm{e}-04$ & $5.62 \mathrm{e}-10$ & $1.16 \mathrm{e}-04$ & $5.62 \mathrm{e}-14$ & $1.16 \mathrm{e}-08$ \\
\hline BY109 & 1600 & $1 \mathrm{e}-04$ & $6.44 \mathrm{e}-10$ & $3.75 \mathrm{e}-0.5$ & $6.44 \mathrm{e}-14$ & $3.75 \mathrm{e}-09$ \\
\hline BY102 & 1290 & $1 e-04$ & $6.49 \mathrm{e}-10$ & $9.88 \mathrm{e}-05$ & $6.49 \mathrm{e}-14$ & $9.88 \mathrm{e}-09$ \\
\hline BY 106 & 2430 & $1 e-04$ & $6.68 \mathrm{e}-10$ & $1.15 \mathrm{e}-04$ & $6.68 \mathrm{e}-14$ & $1.15 \mathrm{e}-08$ \\
\hline AX103 & 424 & $1 \mathrm{e}-04$ & $8.05 e-10$ & $1.84 \mathrm{e}-04$ & $8.05 \mathrm{e}-14$ & $1.84 \mathrm{e}-08$ \\
\hline $\mathrm{U} 102$ & 1420 & $1 \mathrm{e}-04$ & $1.03 \mathrm{e}-09$ & $1.19 \mathrm{e}-04$ & $1.03 e-13$ & $1.19 \mathrm{e}-08$ \\
\hline U106 & 857 & $1 e-04$ & $2.33 \mathrm{e}-09$ & $2.46 \mathrm{e}-04$ & $2.33 \mathrm{e}-13$ & $2.46 e-08$ \\
\hline BY105 & 1910 & $1 \mathrm{e}-04$ & $2.46 \mathrm{e}-09$ & $1.74 \mathrm{e}-04$ & $2.46 \mathrm{e}-13$ & $1.74 \mathrm{e}-08$ \\
\hline A101 & 3610 & $1 \mathrm{e}-04$ & $3.73 \mathrm{e}-09$ & $2.68 \mathrm{e}-0.5$ & $3.73 \mathrm{e}-13$ & $2.68 \mathrm{e}-09$ \\
\hline U111 & 1250 & $1 \mathrm{e}-04$ & $2.61 \mathrm{e}-08$ & $4.90 \mathrm{e}-0.5$ & $2.61 \mathrm{e}-12$ & $4.90 \mathrm{e}-09$ \\
\hline U109 & 1750 & $1 \mathrm{e}-04$ & $7.22 \mathrm{e}-06$ & $1.67 \mathrm{e}-03$ & T.22e-10 & $1.67 \mathrm{e}-07$ \\
\hline $\mathrm{S} 107$ & 1430 & $1 e-04$ & $9.49 \mathrm{e}-05$ & $6.0 T e-03$ & $9.49 \mathrm{e}-09$ & $6.07 \mathrm{e}-07$ \\
\hline $\mathrm{S} 101$ & 1620 & $1 e-04$ & $1.57 \mathrm{e}-04$ & $1.13 \mathrm{e}-02$ & $1.57 \mathrm{e}-08$ & $1.13 \mathrm{e}-06$ \\
\hline$\$ 110$ & 1480 & $1 \mathrm{e}-04$ & $3.57 \mathrm{e}-04$ & $2.18 \mathrm{e}-02$ & $3.57 \mathrm{e}-08$ & $2.18 \mathrm{e}-06$ \\
\hline TX104 & 246 & $1 \mathrm{e}-04$ & $4.19 \mathrm{e}-04$ & $3.18 \mathrm{e}-02$ & $4.19 \mathrm{e}-08$ & $3.18 \mathrm{e}-06$ \\
\hline $\mathrm{S} 108$ & 2290 & $1 \mathrm{e}-04$ & $4.32 \mathrm{e}-04$ & $3.80 \mathrm{e}-02$ & $4.32 \mathrm{e}-08$ & $3.80 \mathrm{e}-06$ \\
\hline $\mathrm{S} 112$ & 1980 & $1 \mathrm{e}-04$ & $4.45 \mathrm{e}-04$ & $3.14 \mathrm{e}-02$ & $4.45 \mathrm{e}-08$ & $3.14 \mathrm{e}-06$ \\
\hline $\mathrm{S} 102$ & 2080 & $1 e-04$ & $4.70 \mathrm{e}-04$ & $3.34 \mathrm{e}-02$ & $4.70 \mathrm{e}-08$ & $3.34 \mathrm{e}-06$ \\
\hline $\mathrm{S} 103$ & 940 & $1 \mathrm{e}-04$ & $5.23 \mathrm{e}-04$ & $2.75 \mathrm{e}-02$ & $5.23 \mathrm{e}-08$ & $2.75 \mathrm{e}-06$ \\
\hline $\mathrm{S} 106$ & 1820 & $1 e-04$ & $5.28 \mathrm{e}-04$ & $3.24 \mathrm{e}-02$ & $5.28 \mathrm{e}-08$ & $3.24 \mathrm{e}-06$ \\
\hline $\mathrm{S} 109$ & 2150 & $1 \mathrm{e}-04$ & $6.03 \mathrm{e}-04$ & $2.90 \mathrm{e}-02$ & $6.03 e-08$ & $2.90 \mathrm{e}-06$ \\
\hline TX107 & 136 & $1 e-04$ & $6.89 \mathrm{e}-04$ & $3.63 \mathrm{e}-02$ & $6.89 \mathrm{e}-08$ & $3.63 \mathrm{e}-06$ \\
\hline U108 & 1770 & $1 e-04$ & $1.66 \mathrm{e}-03$ & $5.53 \mathrm{e}-02$ & $1.66 \mathrm{e}-07$ & $5.53 \mathrm{e}-06$ \\
\hline $\mathrm{U} 107$ & 1540 & $1 e-04$ & $2.04 \mathrm{e}-03$ & $6.97 \mathrm{e}-02$ & $2.04 \mathrm{e}-07$ & $6.97 \mathrm{e}-06$ \\
\hline ¿105 & 1580 & $1 e-04$ & $2.15 \mathrm{e}-03$ & $4.42 \mathrm{e}-02$ & $2.15 \mathrm{e}-07$ & $4.42 \mathrm{e}-06$ \\
\hline BY 108 & 864 & $1 e-04$ & $2.92 \mathrm{e}-03$ & $3.34 \mathrm{e}-02$ & $2.92 \mathrm{e}-07$ & $3.34 \mathrm{e}-06$ \\
\hline TX109 & 1460 & $1 \mathrm{e}-04$ & $4.26 \mathrm{e}-03$ & $8.68 \mathrm{e}-02$ & $4.26 \mathrm{e}-07$ & $8.68 \mathrm{e}-06$ \\
\hline TX112 & 2460 & $1 \mathrm{e}-04$ & $4.80 \mathrm{e}-03$ & $8.46 \mathrm{e}-02$ & $4.80 \mathrm{e}-0 \mathrm{~T}$ & $8.46 e-06$ \\
\hline
\end{tabular}


Table 2: Probability Calculations for Reactive Region 1

\begin{tabular}{|rr|ccc|cc|}
\hline & Vol & Fail & \multicolumn{2}{c|}{ React. Waste } & \multicolumn{2}{c|}{ Prob Fire } \\
Tank & $M^{3}$ & Prob & Median & Upper-95\% & Median & Upper-95\% \\
\hline S111 & 2260 & $1 \mathrm{e}-04$ & $4.91 \mathrm{e}-03$ & $4.77 \mathrm{e}-02$ & $4.91 \mathrm{e}-07$ & $4.7 \mathrm{Te}-06$ \\
TX110 & 1750 & $1 \mathrm{e}-04$ & $5.02 \mathrm{e}-03$ & $8.48 \mathrm{e}-02$ & $5.02 \mathrm{e}-07$ & $8.48 \mathrm{e}-06$ \\
TX113 & 2300 & $1 \mathrm{e}-04$ & $5.08 \mathrm{e}-03$ & $8.89 \mathrm{e}-02$ & $5.08 \mathrm{e}-07$ & $8.89 \mathrm{e}-06$ \\
TX117 & 2370 & $1 \mathrm{e}-04$ & $5.16 \mathrm{e}-03$ & $8.07 \mathrm{e}-02$ & $5.16 \mathrm{e}-07$ & $8.07 \mathrm{e}-06$ \\
TX116 & 2390 & $1 \mathrm{e}-04$ & $5.26 \mathrm{e}-03$ & $8.29 \mathrm{e}-02$ & $5.26 \mathrm{e}-07$ & $8.29 \mathrm{e}-06$ \\
TX114 & 2030 & $1 \mathrm{e}-04$ & $6.05 \mathrm{e}-03$ & $8.10 \mathrm{e}-02$ & $6.05 \mathrm{e}-07$ & $8.10 \mathrm{e}-06$ \\
TX111 & 1400 & $1 \mathrm{e}-04$ & $6.10 \mathrm{e}-03$ & $8.87 \mathrm{e}-02$ & $6.10 \mathrm{e}-07$ & $8.87 \mathrm{e}-06$ \\
BY110 & 1510 & $1 \mathrm{e}-04$ & $7.16 \mathrm{e}-03$ & $8.21 \mathrm{e}-02$ & $7.16 \mathrm{e}-07$ & $8.21 \mathrm{e}-06$ \\
SX102 & 2060 & $1 \mathrm{e}-04$ & 0.00721 & 0.0610 & $7.21 \mathrm{e}-07$ & $6.10 \mathrm{e}-06$ \\
SX101 & 1730 & $1 \mathrm{e}-04$ & 0.00735 & 0.0652 & $7.35 \mathrm{e}-07$ & $6.52 \mathrm{e}-06$ \\
BY107 & 1010 & $1 \mathrm{e}-04$ & 0.00747 & 0.0900 & $7.47 \mathrm{e}-07$ & $9.00 \mathrm{e}-06$ \\
SX103 & 2470 & $1 \mathrm{e}-04$ & 0.00778 & 0.0729 & $7.78 \mathrm{e}-07$ & $7.29 \mathrm{e}-06$ \\
SX106 & 2040 & $1 \mathrm{e}-04$ & 0.00780 & 0.0710 & $7.80 \mathrm{e}-07$ & $7.10 \mathrm{e}-06$ \\
BY101 & 1470 & $1 \mathrm{e}-04$ & 0.00823 & 0.0817 & $8.23 \mathrm{e}-07$ & $8.17 \mathrm{e}-06$ \\
TX108 & 508 & $1 \mathrm{e}-04$ & 0.00825 & 0.0978 & $8.25 \mathrm{e}-07$ & $9.78 \mathrm{e}-06$ \\
BY112 & 1100 & $1 \mathrm{e}-04$ & 0.00828 & 0.1050 & $8.28 \mathrm{e}-07$ & $1.05 \mathrm{e}-05$ \\
SX104 & 2330 & $1 \mathrm{e}-04$ & 0.00846 & 0.0642 & $8.46 \mathrm{e}-07$ & $6.42 \mathrm{e}-06$ \\
TX118 & 1320 & $1 \mathrm{e}-04$ & 0.00865 & 0.0990 & $8.65 \mathrm{e}-07$ & $9.90 \mathrm{e}-06$ \\
SX105 & 2590 & $1 \mathrm{e}-04$ & 0.00905 & 0.0763 & $9.05 \mathrm{e}-07$ & $7.63 \mathrm{e}-06$ \\
TX115 & 2430 & $1 \mathrm{e}-04$ & 0.00917 & 0.1040 & $9.17 \mathrm{e}-07$ & $1.04 \mathrm{e}-05$ \\
BY111 & 1740 & $1 \mathrm{e}-04$ & 0.01030 & 0.1070 & $1.03 \mathrm{e}-06$ & $1.07 \mathrm{e}-05$ \\
TX106 & 1720 & $1 \mathrm{e}-04$ & 0.01210 & 0.0916 & $1.21 \mathrm{e}-06$ & $9.16 \mathrm{e}-06$ \\
TX105 & 2310 & $1 \mathrm{e}-04$ & 0.01280 & 0.0936 & $1.28 \mathrm{e}-06$ & $9.36 \mathrm{e}-06$ \\
S105 & 1730 & $1 \mathrm{e}-04$ & 0.01290 & 0.0867 & $1.29 \mathrm{e}-06$ & $8.67 \mathrm{e}-06$ \\
TX102 & 822 & $1 \mathrm{e}-04$ & 0.01300 & 0.0930 & $1.30 \mathrm{e}-06$ & $9.30 \mathrm{e}-06$ \\
BY104 & 1540 & $1 \mathrm{e}-04$ & 0.01590 & 0.0937 & $1.59 \mathrm{e}-06$ & $9.37 \mathrm{e}-06$ \\
\hline
\end{tabular}


Table 3: Probability Calculations for Reactive Region 2

\begin{tabular}{|c|c|c|c|c|c|c|}
\hline Tank & $\begin{array}{c}\mathrm{N} \\
\mathrm{Obs}\end{array}$ & $\operatorname{Pr}$ (fail) & $\begin{array}{l}\text { Median } \\
\mathrm{R} \text { Waste }\end{array}$ & $\begin{array}{l}\text { Upper } 95 \\
\text { R Waste }\end{array}$ & $\begin{array}{l}\text { Median } \\
\operatorname{Pr}(\text { fire })\end{array}$ & $\begin{array}{c}\text { Upper } 95 \\
\text { Pr(fire) }\end{array}$ \\
\hline$\overline{\mathrm{BX} 110}$ & 0 & $1.00 \mathrm{e}-04$ & $3.02 \mathrm{e}-05$ & $1.14 \mathrm{e}-03$ & $3.02 \mathrm{e}-09$ & $1.14 \mathrm{e}-07$ \\
\hline BX105 & 0 & $1.00 \mathrm{e}-04$ & $1.80 \mathrm{e}-04$ & $4.35 \mathrm{e}-03$ & $1.80 \mathrm{e}-08$ & $4.35 \mathrm{e}-07$ \\
\hline TY102 & 1 & $1.00 \mathrm{e}-04$ & $2.11 \mathrm{e}-04$ & $1.22 \mathrm{e}-02$ & $2.11 \mathrm{e}-08$ & $1.22 \mathrm{e}-06$ \\
\hline B104 & 0 & $1.00 \mathrm{e}-04$ & $6.35 \mathrm{e}-04$ & $1.50 e-02$ & $6.35 e-08$ & $1.50 \mathrm{e}-06$ \\
\hline $\mathrm{B} 10^{\circ}$ & 0 & $1.00 \mathrm{e}-04$ & $9.97 \mathrm{e}-04$ & $2.44 \mathrm{e}-02$ & $9.97 \mathrm{e}-08$ & $2.44 \mathrm{e}-06$ \\
\hline A 102 & 0 & $1.00 \mathrm{e}-04$ & $1.73 \mathrm{e}-03$ & $4.22 \mathrm{e}-02$ & $1.73 \mathrm{e}-07$ & $4.22 \mathrm{e}-06$ \\
\hline $\mathrm{AX} 102$ & 0 & $1.00 \mathrm{e}-04$ & $2.01 e-03$ & $5.30 e-02$ & $2.01 \mathrm{e}-07$ & $5.30 e-06$ \\
\hline BX111 & 0 & 1.00 & $2.07 \mathrm{e}-03$ & $3.95 e-02$ & $2.07 \mathrm{e}-07$ & $3.95 \mathrm{e}-06$ \\
\hline B105 & 0 & $1.00 \mathrm{e}-04$ & $2.11 \mathrm{e}-03$ & $6.34 \mathrm{e}-02$ & $2.11 \mathrm{e}-07$ & $6.34 \mathrm{e}-06$ \\
\hline BY109 & 0 & 1.00 & $2.29 \mathrm{e}-03$ & $6.54 \mathrm{e}-02$ & $2.29 \mathrm{e}-07$ & 6.5 \\
\hline BY 102 & 0 & $1.00 \mathrm{e}-04$ & $3.49 \mathrm{e}-03$ & Т.47e-02 & $3.49 \mathrm{e}-07$ & T.47e-06 \\
\hline AX101 & 0 & 1.0 & $3.51 \mathrm{e}-03$ & $8.87 \epsilon$ & $3.51 \mathrm{e}-07$ & 8.8 \\
\hline $\mathrm{AX} 103$ & 0 & 1.00 & $3.72 \mathrm{e}$ & 7.62 & $3.72 \mathrm{e}-07$ & 7.6 \\
\hline BY 105 & 0 & 1.00 & $3.89 \mathrm{e}-03$ & $8.10 \mathrm{e}-02$ & $3.89 \mathrm{e}-07$ & 8.10 \\
\hline U103 & 1 & & & & & 06 \\
\hline U106 & 0 & $1.00 \mathrm{e}-04$ & $4.21 \mathrm{e}-03$ & $6.70 \mathrm{e}-02$ & $4.21 \mathrm{e}-07$ & $6.70 \mathrm{e}-06$ \\
\hline & 0 & $1.00 \mathrm{e}-04$ & $4.35 \mathrm{e}-03$ & $8.29 \mathrm{e}-02$ & $4.35 e-07$ & 8.2 \\
\hline U102 & 0 & $1.00 \mathrm{e}-04$ & $4.38 \mathrm{e}-03$ & $8.32 \mathrm{e}-02$ & $4.38 \mathrm{e}-07$ & $8.32 \mathrm{e}-06$ \\
\hline S107 & 0 & 1.00 & $4.41 \mathrm{e}-03$ & $2.39 e-02$ & $4.41 \mathrm{e}-07$ & 2.3 \\
\hline BY103 & 0 & $1.00 \mathrm{e}-04$ & $5.18 \mathrm{e}-03$ & $8.73 \mathrm{e}-02$ & $5.18 \mathrm{e}-07$ & $8.73 e-06$ \\
\hline A101 & 2 & 1.00 & $9.04 \mathrm{e}-03$ & $8.62 \mathrm{e}-02$ & $9.04 \mathrm{e}-07$ & $8.62 \mathrm{e}-06$ \\
\hline S101 & 0 & 1.00 & $9.76 \mathrm{e}-03$ & $5.11 \mathrm{e}-02$ & $9.76 \mathrm{e}-07$ & 5.1 \\
\hline $\mathrm{S} 110$ & 0 & 1.0 & $1.40 \mathrm{e}-02$ & $8.23 e-02$ & $1.40 \mathrm{e}-06$ & 8.23 \\
\hline U111 & 2 & 1.0 & $1.53 \mathrm{e}-02$ & $8.75 e-02$ & $1.53 \mathrm{e}-06$ & 8.7 \\
\hline BY108 & 0 & 1.0 & $1.79 \mathrm{e}-02$ & $6.41 e$ & $1.79 \mathrm{e}-06$ & 6.41 \\
\hline S103 & 0 & $1.00 \mathrm{e}-04$ & $1.91 \mathrm{e}-02$ & $1.03 \mathrm{e}-01$ & $1.91 \mathrm{e}-06$ & $1.03 \mathrm{e}-05$ \\
\hline $\mathrm{S} 11.1$ & 3 & 1.00 & $1.93 \mathrm{e}-02$ & $9.14 \mathrm{e}-02$ & $1.93 \mathrm{e}-06$ & $9.14 \mathrm{e}-06$ \\
\hline $\mathrm{SX} 102$ & 2 & $1.00 \mathrm{e}-04$ & $2.00 \mathrm{e}-02$ & $1.02 \mathrm{e}-01$ & $2.00 \mathrm{e}-06$ & $1.02 \mathrm{e}-05$ \\
\hline TX107 & 0 & $1.00 \mathrm{e}$ & $2.00 \mathrm{e}-02$ & $1.11 \mathrm{e}-01$ & $2.00 \mathrm{e}-06$ & $1.11 \mathrm{e}-05$ \\
\hline SX101 & 0 & $1.00 \mathrm{e}-04$ & $2.05 e-02$ & $1.06 \mathrm{e}-01$ & $2.05 e-06$ & $1.06 \mathrm{e}-05$ \\
\hline S106 & 0 & $1.00 \mathrm{e}-04$ & $2.17 \mathrm{e}-02$ & $1.09 \mathrm{e}-01$ & $2.17 \mathrm{e}-06$ & $1.09 \mathrm{e}-05$ \\
\hline SX104 & 0 & $1.00 \mathrm{e}-04$ & $2.19 \mathrm{e}-02$ & $1.13 \mathrm{e}-01$ & $2.19 \mathrm{e}-06$ & $1.13 e-05$ \\
\hline SX103 & 1 & $1.00 \mathrm{e}-04$ & $2.23 \mathrm{e}-02$ & $1.06 \mathrm{e}-01$ & $2.23 \mathrm{e}-06$ & $1.06 \mathrm{e}-05$ \\
\hline SX106 & 0 & $1.00 \mathrm{e}-04$ & $2.25 \mathrm{e}-02$ & $1.12 \mathrm{e}-01$ & $2.25 \mathrm{e}-06$ & $1.12 \mathrm{e}-05$ \\
\hline TX104 & 0 & $1.00 \mathrm{e}-04$ & $2.27 \mathrm{e}-02$ & $1.23 \mathrm{e}-01$ & $2.27 e-06$ & $1.23 \mathrm{e}-05$ \\
\hline $\mathrm{S} 109$ & 1 & $1.00 \mathrm{e}-04$ & $2.33 \mathrm{e}-02$ & $1.26 \mathrm{e}-01$ & $2.33 \mathrm{e}-06$ & $1.26 \mathrm{e}-05$ \\
\hline $\mathrm{S} 108$ & 0 & $1.00 \mathrm{e}-04$ & $2.33 \mathrm{e}-02$ & $1.22 \mathrm{e}-01$ & $2.33 \mathrm{e}-06$ & $1.22 \mathrm{e}-05$ \\
\hline SX105 & 0 & $1.00 \mathrm{e}-04$ & $2.40 \mathrm{e}-02$ & $1.15 \mathrm{e}-01$ & $2.40 \mathrm{e}-06$ & $1.15 \mathrm{e}-05$ \\
\hline $\mathrm{S} 102$ & 0 & $1.00 \mathrm{e}-04$ & $2.42 \mathrm{e}-02$ & $1.28 \mathrm{e}-01$ & $2.42 e-06$ & $1.28 \mathrm{e}-0.5$ \\
\hline
\end{tabular}


Table 3: Probability Calculations for Reactive Region 2

\begin{tabular}{|c|c|c|c|c|c|c|}
\hline Tank & $\begin{array}{c}\mathrm{N} \\
\text { Obs }\end{array}$ & $\operatorname{Pr}($ fail $)$ & $\begin{array}{c}\text { Median } \\
\text { R Waste } \\
\end{array}$ & $\begin{array}{l}\text { Upper } 95 \\
\text { R Waste } \\
\end{array}$ & $\begin{array}{l}\text { Median } \\
\operatorname{Pr}(\text { fire }) \\
\end{array}$ & $\begin{array}{l}\text { Upper } 95 \\
\operatorname{Pr} \text { (fire) } \\
\end{array}$ \\
\hline S112 & 0 & $1.00 \mathrm{e}-04$ & $2.43 \mathrm{e}-02$ & $1.18 \mathrm{e}-01$ & $2.43 \mathrm{e}-06$ & $1.18 \mathrm{e}-05$ \\
\hline U109 & 0 & $1.00 \mathrm{e}-04$ & $3.27 \mathrm{e}-02$ & 1. $47 \mathrm{e}-01$ & $3.2 \tau \mathrm{e}-06$ & $1.47 \mathrm{e}-0.5$ \\
\hline TX102 & 1 & $1.00 \mathrm{e}-04$ & $3.39 \mathrm{e}-02$ & $1.46 \mathrm{e}-01$ & $3.39 \mathrm{e}-06$ & $1.46 \mathrm{e}-0.5$ \\
\hline TX111 & 0 & $1.00 \mathrm{e}-04$ & $3.41 \mathrm{e}-02$ & 1.70e-01 & $3.41 \mathrm{e}-06$ & $1.70 \mathrm{e}-05$ \\
\hline TX117 & 0 & $1.00 \mathrm{e}-04$ & $3.57 \mathrm{e}-02$ & $1.77 \mathrm{e}-01$ & $3.57 \mathrm{e}-06$ & $1.77 \mathrm{e}-05$ \\
\hline TX112 & 0 & $1.00 \mathrm{e}-04$ & $3.63 \mathrm{e}-02$ & $1.81 \mathrm{e}-01$ & $3.63 \mathrm{e}-06$ & $1.81 \mathrm{e}-05$ \\
\hline TX106 & 0 & $1.00 \mathrm{e}-04$ & $3.63 \mathrm{e}-02$ & $1.51 \mathrm{e}-01$ & $3.63 \mathrm{e}-06$ & $1.51 \mathrm{e}-05$ \\
\hline TX109 & 0 & $1.00 \mathrm{e}-04$ & $3.65 \mathrm{e}-02$ & $1.85 \mathrm{e}-01$ & $3.65 \mathrm{e}-06$ & $1.85 \mathrm{e}-05$ \\
\hline TX114 & 0 & $1.00 \mathrm{e}-04$ & $3.71 \mathrm{e}-02$ & $1.68 \mathrm{e}-01$ & $3.71 \mathrm{e}-06$ & $1.68 \mathrm{e}-05$ \\
\hline TX113 & 0 & $1.00 \mathrm{e}-04$ & $3.73 \mathrm{e}-02$ & $1.72 \mathrm{e}-01$ & $3.73 \mathrm{e}-06$ & $1.72 \mathrm{e}-05$ \\
\hline S105 & 0 & $1.00 \mathrm{e}-04$ & $3.75 \mathrm{e}-02$ & $1.53 \mathrm{e}-01$ & $3.75 \mathrm{e}-06$ & $1.53 \mathrm{e}-05$ \\
\hline TX105 & 0 & $1.00 \mathrm{e}-04$ & $3.78 \mathrm{e}-02$ & $1.56 \mathrm{e}-01$ & $3.78 \mathrm{e}-06$ & $1.56 \mathrm{e}-05$ \\
\hline TX116 & 0 & $1.00 \mathrm{e}-04$ & $3.91 \mathrm{e}-02$ & $1.77 \mathrm{e}-01$ & $3.91 \mathrm{e}-06$ & $1.77 \mathrm{e}-05$ \\
\hline BY101 & 0 & $1.00 \mathrm{e}-04$ & $4.02 \mathrm{e}-02$ & $1.37 \mathrm{e}-01$ & $4.02 \mathrm{e}-06$ & $1.37 \mathrm{e}-0.5$ \\
\hline TX110 & 0 & $1.00 \mathrm{e}-04$ & $4.07 \mathrm{e}-02$ & $1.74 \mathrm{e}-01$ & $4.07 \mathrm{e}-06$ & $1.74 \mathrm{e}-05$ \\
\hline BY110 & 0 & $1.00 \mathrm{e}-04$ & $4.08 \mathrm{e}-02$ & $1.57 \mathrm{e}-01$ & $4.08 \mathrm{e}-06$ & $1.57 \mathrm{e}-05$ \\
\hline BY107 & 0 & $1.00 \mathrm{e}-04$ & $4.36 \mathrm{e}-02$ & $1.49 \mathrm{e}-01$ & $4.36 \mathrm{e}-06$ & $1.49 \mathrm{e}-0.5$ \\
\hline U107 & 0 & $1.00 \mathrm{e}-04$ & $4.90 \mathrm{e}-02$ & $1.90 \mathrm{e}-01$ & $4.90 \mathrm{e}-06$ & $1.90 \mathrm{e}-0.5$ \\
\hline U108 & 0 & $1.00 \mathrm{e}-04$ & $4.95 \mathrm{e}-02$ & $1.92 \mathrm{e}-01$ & $4.95 \mathrm{e}-06$ & $1.92 \mathrm{e}-05$ \\
\hline U105 & 1 & $1.00 \mathrm{e}-04$ & $4.98 \mathrm{e}-02$ & $1.76 \mathrm{e}-01$ & $4.98 \mathrm{e}-06$ & $1.76 \mathrm{e}-05$ \\
\hline TX118 & 1 & $1.00 \mathrm{e}-04$ & $5.14 \mathrm{e}-02$ & $1.98 \mathrm{e}-01$ & $5.14 \mathrm{e}-06$ & $1.98 \mathrm{e}-05$ \\
\hline BY104 & 4 & $1.00 \mathrm{e}-04$ & $5.47 \mathrm{e}-02$ & $1.91 \mathrm{e}-01$ & $5.47 \mathrm{e}-06$ & $1.91 \mathrm{e}-05$ \\
\hline TX108 & 0 & $1.00 \mathrm{e}-04$ & $5.50 \mathrm{e}-02$ & $1.99 \mathrm{e}-01$ & $5.50 \mathrm{e}-06$ & $1.99 \mathrm{e}-05$ \\
\hline BY112 & 0 & $1.00 \mathrm{e}-04$ & $5.62 \mathrm{e}-02$ & $2.03 \mathrm{e}-01$ & $5.62 \mathrm{e}-06$ & $2.03 \mathrm{e}-05$ \\
\hline TX115 & 0 & $1.00 \mathrm{e}-04$ & $5.64 \mathrm{e}-02$ & $2.07 \mathrm{e}-01$ & $5.64 \mathrm{e}-06$ & $2.07 \mathrm{e}-05$ \\
\hline BY111 & 0 & $1.00 \mathrm{e}-04$ & $5.66 \mathrm{e}-02$ & $1.95 \mathrm{e}-01$ & $5.66 \mathrm{e}-06$ & $1.95 \mathrm{e}-05$ \\
\hline
\end{tabular}


Table 4: Probability Calculations for Reactive Region 3

\begin{tabular}{|c|c|c|c|c|c|c|}
\hline Tank & $\begin{array}{c}\mathrm{N} \\
\mathrm{Obs}\end{array}$ & $\operatorname{Pr}($ fail $)$ & $\begin{array}{l}\text { Median } \\
\mathrm{R} \text { Waste }\end{array}$ & $\begin{array}{l}\text { Upper } 95 \\
\mathrm{R} \text { Waste }\end{array}$ & $\begin{array}{l}\text { Median } \\
\text { Pr(fire) }\end{array}$ & $\begin{array}{c}\text { Upper } 95 \\
\text { Pr(fire) }\end{array}$ \\
\hline BX110 & 0 & $1.00 \mathrm{e}-04$ & $0.00 \mathrm{e}+00$ & $2.90 \mathrm{e}-07$ & $0.00 \mathrm{e}+00$ & $2.90 \mathrm{e}-11$ \\
\hline TY 102 & 1 & $1.00 \mathrm{e}-04$ & 0 & $1.13 e-06$ & $0.00 \mathrm{e}+00$ & $1.13 \mathrm{e}-10$ \\
\hline BX105 & 0 & $1.00 \mathrm{e}-04$ & $1.49 \mathrm{e}-10$ & $1.7 \mathrm{Se}-05$ & $1.49 \mathrm{e}-14$ & $1.78 \mathrm{e}-09$ \\
\hline B102 & 0 & $1.00 \mathrm{e}-04$ & $2.90 \mathrm{e}-10$ & $7.00 \mathrm{e}-05$ & $2.90 \mathrm{e}-14$ & $7.00 \mathrm{e}-09$ \\
\hline B104 & 0 & $1.00 \mathrm{e}-04$ & $3.27 \mathrm{e}-10$ & $4.04 \mathrm{e}-05$ & $3.27 \mathrm{e}-14$ & $4.04 \mathrm{e}-09$ \\
\hline $\mathrm{A} 102$ & 0 & $1.00 \mathrm{e}-04$ & $7.59 \mathrm{e}-10$ & $1.20 \mathrm{e}-04$ & $7.59 \mathrm{e}-14$ & $1.20 \mathrm{e}-08$ \\
\hline BY109 & 0 & $1.00 \mathrm{e}-04$ & $1.15 \mathrm{e}-09$ & $1.09 \mathrm{e}-04$ & $1.15 \mathrm{e}-13$ & $1.09 \mathrm{e}-08$ \\
\hline AX101 & 0 & $1.00 \mathrm{e}-04$ & $1.44 \mathrm{e}-09$ & $2.89 \mathrm{e}-04$ & $1.44 \mathrm{e}-13$ & $2.89 \mathrm{e}-08$ \\
\hline U102 & 0 & $1.00 \mathrm{e}-04$ & $1.48 \mathrm{e}-09$ & $2.12 \mathrm{e}-04$ & $1.48 \mathrm{e}-13$ & $2.12 \mathrm{e}-08$ \\
\hline BX111 & 0 & $1.00 \mathrm{e}-04$ & $1.54 \mathrm{e}-09$ & $7.88 \mathrm{e}-05$ & $1.54 \mathrm{e}-13$ & $7.88 \mathrm{e}-09$ \\
\hline BY 106 & 0 & $1.00 \mathrm{e}-04$ & $1.84 \mathrm{e}-09$ & $1.49 \mathrm{e}-04$ & $1.84 \mathrm{e}-13$ & $1.49 \mathrm{e}-08$ \\
\hline BY 102 & 0 & $1.00 \mathrm{e}-04$ & $1.95 \mathrm{e}-09$ & $2.00 \mathrm{e}-04$ & $1.95 \mathrm{e}-13$ & $2.00 \mathrm{e}-08$ \\
\hline U106 & 0 & $1.00 \mathrm{e}-04$ & $2.16 \mathrm{e}-09$ & $1.90 \mathrm{e}-04$ & $2.16 \mathrm{e}-13$ & $1.90 \mathrm{e}-08$ \\
\hline $\mathrm{AX} 102$ & 0 & $1.00 \mathrm{e}-04$ & $2.35 \mathrm{e}-09$ & $1.09 \mathrm{e}-04$ & $2.35 \mathrm{e}-13$ & $1.09 \mathrm{e}-08$ \\
\hline U103 & 1 & $1.00 \mathrm{e}-04$ & $2.39 \mathrm{e}-09$ & $2.27 \mathrm{e}-04$ & $2.39 \mathrm{e}-13$ & $2.27 \mathrm{e}-08$ \\
\hline B105 & 0 & $1.00 \mathrm{e}-04$ & $2.54 \mathrm{e}-09$ & $1.58 \mathrm{e}-04$ & $2.54 \mathrm{e}-13$ & $1.58 \mathrm{e}-08$ \\
\hline AX103 & 0 & $1.00 \mathrm{e}-04$ & $2.80 \mathrm{e}-09$ & $1.35 \mathrm{e}-04$ & $2.80 \mathrm{e}-13$ & $1.35 e-08$ \\
\hline BY 105 & 0 & $1.00 \mathrm{e}-04$ & $2.91 \mathrm{e}-09$ & $3.77 \mathrm{e}-04$ & $2.91 \mathrm{e}-13$ & $3.77 \mathrm{e}-08$ \\
\hline BY103 & 0 & $1.00 \mathrm{e}-04$ & $2.93 \mathrm{e}-09$ & $3.03 \mathrm{e}-04$ & $2.93 \mathrm{e}-13$ & $3.03 e-08$ \\
\hline A101. & 2 & $1.00 \mathrm{e}-04$ & $2.64 \mathrm{e}-08$ & $6.87 \mathrm{e}-05$ & $2.64 \mathrm{e}-12$ & $6.87 e-09$ \\
\hline U111 & 2 & $1.00 \mathrm{e}-04$ & $1.40 \mathrm{e}-07$ & $1.43 \mathrm{e}-04$ & $1.40 \mathrm{e}-11$ & $1.43 \mathrm{e}-08$ \\
\hline U109 & 0 & $1.00 \mathrm{e}-04$ & $2.57 \mathrm{e}-05$ & $2.97 \mathrm{e}-03$ & $2.57 \mathrm{e}-09$ & $2.97 \mathrm{e}-07$ \\
\hline S10T & 0 & $1.00 \mathrm{e}-04$ & $2.63 \mathrm{e}-04$ & $1.10 \mathrm{e}-02$ & $2.63 e-08$ & $1.10 \mathrm{e}-06$ \\
\hline S101 & 0 & $1.00 \mathrm{e}-04$ & $6.76 \mathrm{e}-04$ & $1.83 \mathrm{e}-02$ & $6.76 \mathrm{e}-08$ & e-06 \\
\hline $\mathrm{S} 110$ & 0 & $1.00 \mathrm{e}-04$ & $7.87 e-04$ & $3.05 \mathrm{e}-02$ & $7.87 \mathrm{e}-08$ & $3.05 e-06$ \\
\hline$\$ 108$ & 0 & $1.00 \mathrm{e}-04$ & $1.14 \mathrm{e}-03$ & $4.67 \mathrm{e}-02$ & $1.14 \mathrm{e}-07$ & $4.67 \mathrm{e}-06$ \\
\hline S106 & 0 & $1.00 \mathrm{e}-04$ & $1.24 \mathrm{e}-03$ & $4.21 \mathrm{e}-02$ & $1.24 \mathrm{e}-07$ & $4.21 \mathrm{e}-06$ \\
\hline $\mathrm{S} 112$ & 0 & $1.00 \mathrm{e}-04$ & $1.29 \mathrm{e}-03$ & $5.44 \mathrm{e}-02$ & $1.29 \mathrm{e}-07$ & $5.44 \mathrm{e}-06$ \\
\hline TX104 & 0 & $1.00 \mathrm{e}-04$ & $1.30 \mathrm{e}-03$ & $4.81 \mathrm{e}-02$ & $1.30 \mathrm{e}-07$ & $4.81 \mathrm{e}-06$ \\
\hline $\mathrm{S} 103$ & 0 & $1.00 \mathrm{e}-04$ & $1.33 \mathrm{e}-03$ & $4.50 \mathrm{e}-02$ & $1.33 \mathrm{e}-07$ & $4.50 \mathrm{e}-06$ \\
\hline S109 & 1 & $1.00 \mathrm{e}-04$ & $1.50 \mathrm{e}-03$ & $4.92 \mathrm{e}-02$ & $1.50 \mathrm{e}-07$ & $4.92 \mathrm{e}-06$ \\
\hline $\mathrm{S} 102$ & 0 & $1.00 \mathrm{e}-04$ & $1.58 \mathrm{e}-03$ & $4.66 \mathrm{e}-02$ & $1.58 \mathrm{e}-07$ & $4.66 \mathrm{e}-06$ \\
\hline TX107 & 0 & $1.00 \mathrm{e}-04$ & $1.65 \mathrm{e}-03$ & $5.53 \mathrm{e}-02$ & $1.65 \mathrm{e}-07$ & $5.53 \mathrm{e}-06$ \\
\hline U107 & 0 & $1.00 \mathrm{e}-04$ & $4.11 \mathrm{e}-03$ & $8.39 \mathrm{e}-02$ & $4.11 \mathrm{e}-07$ & $8.39 \mathrm{e}-06$ \\
\hline 0108 & 0 & $1.00 \mathrm{e}-04$ & $4.22 \mathrm{e}-03$ & $8.82 \mathrm{e}-02$ & $4.22 \mathrm{e}-07$ & $8.82 \mathrm{e}-06$ \\
\hline U105 & 1 & $1.00 \mathrm{e}-04$ & $5.87 \mathrm{e}-03$ & $6.70 \mathrm{e}-02$ & $5.87 \mathrm{e}-07$ & $6.70 \mathrm{e}-06$ \\
\hline BY108 & 0 & $1.00 \mathrm{e}-04$ & 7.35e-03 & $4.86 \mathrm{e}-02$ & $7.35 \mathrm{e}-07$ & $4.86 \mathrm{e}-06$ \\
\hline TX113 & 0 & $1.00 \mathrm{e}-04$ & $1.03 \mathrm{e}-02$ & $1.26 \mathrm{e}-01$ & $1.03 \mathrm{e}-06$ & $1.26 \mathrm{e}-05$ \\
\hline TX117 & 0 & $1.00 \mathrm{e}-04$ & $1.09 \mathrm{e}-02$ & $1.28 \mathrm{e}-01$ & $1.09 \mathrm{e}-06$ & $1.28 \mathrm{e}-05$ \\
\hline
\end{tabular}


Table 4: Probability Calculations for Reactive Region 3

\begin{tabular}{|l||c|c|c|c|c|c|}
\hline Tank & $\begin{array}{c}\text { N } \\
\text { Obs }\end{array}$ & Pr(fail) & $\begin{array}{c}\text { Median } \\
\text { R Waste }\end{array}$ & $\begin{array}{c}\text { Upper } 95 \\
\text { R Waste }\end{array}$ & $\begin{array}{c}\text { Median } \\
\operatorname{Pr}(\text { fire) }\end{array}$ & $\begin{array}{c}\text { Upper } 95 \\
\text { Pr(fire) }\end{array}$ \\
\hline TX116 & 0 & $1.00 \mathrm{e}-04$ & $1.10 \mathrm{e}-02$ & $1.30 \mathrm{e}-01$ & $1.10 \mathrm{e}-06$ & $1.30 \mathrm{e}-05$ \\
TX112 & 0 & $1.00 \mathrm{e}-04$ & $1.23 \mathrm{e}-02$ & $1.29 \mathrm{e}-01$ & $1.23 \mathrm{e}-06$ & $1.29 \mathrm{e}-05$ \\
TX114 & 0 & $1.00 \mathrm{e}-04$ & $1.29 \mathrm{e}-02$ & $1.38 \mathrm{e}-01$ & $1.29 \mathrm{e}-06$ & $1.38 \mathrm{e}-05$ \\
TX110 & 0 & $1.00 \mathrm{e}-04$ & $1.30 \mathrm{e}-02$ & $1.26 \mathrm{e}-01$ & $1.30 \mathrm{e}-06$ & $1.26 \mathrm{e}-05$ \\
S111 & 3 & $1.00 \mathrm{e}-04$ & $1.31 \mathrm{e}-02$ & $7.34 \mathrm{e}-02$ & $1.31 \mathrm{e}-06$ & $7.34 \mathrm{e}-06$ \\
TX109 & 0 & $1.00 \mathrm{e}-04$ & $1.38 \mathrm{e}-02$ & $1.16 \mathrm{e}-01$ & $1.38 \mathrm{e}-06$ & $1.16 \mathrm{e}-05$ \\
TX111 & 0 & $1.00 \mathrm{e}-04$ & $1.39 \mathrm{e}-02$ & $1.34 \mathrm{e}-01$ & $1.39 \mathrm{e}-06$ & $1.34 \mathrm{e}-05$ \\
BY101 & 0 & $1.00 \mathrm{e}-04$ & $1.42 \mathrm{e}-02$ & $1.17 \mathrm{e}-01$ & $1.42 \mathrm{e}-06$ & $1.17 \mathrm{e}-05$ \\
TX118 & 1 & $1.00 \mathrm{e}-04$ & $1.49 \mathrm{e}-02$ & $1.39 \mathrm{e}-01$ & $1.49 \mathrm{e}-06$ & $1.39 \mathrm{e}-05$ \\
BY110 & 0 & $1.00 \mathrm{e}-04$ & $1.57 \mathrm{e}-02$ & $1.15 \mathrm{e}-01$ & $1.57 \mathrm{e}-06$ & $1.15 \mathrm{e}-05$ \\
SX101 & 0 & $1.00 \mathrm{e}-04$ & $1.64 \mathrm{e}-02$ & $8.53 \mathrm{e}-02$ & $1.64 \mathrm{e}-06$ & $8.53 \mathrm{e}-06$ \\
TX108 & 0 & $1.00 \mathrm{e}-04$ & $1.69 \mathrm{e}-02$ & $1.49 \mathrm{e}-01$ & $1.69 \mathrm{e}-06$ & $1.49 \mathrm{e}-05$ \\
SX102 & 2 & $1.00 \mathrm{e}-04$ & $1.75 \mathrm{e}-02$ & $9.45 \mathrm{e}-02$ & $1.75 \mathrm{e}-06$ & $9.45 \mathrm{e}-06$ \\
BY107 & 0 & $1.00 \mathrm{e}-04$ & $1.80 \mathrm{e}-02$ & $1.19 \mathrm{e}-01$ & $1.80 \mathrm{e}-06$ & $1.19 \mathrm{e}-05$ \\
SX106 & 0 & $1.00 \mathrm{e}-04$ & $1.81 \mathrm{e}-02$ & $1.00 \mathrm{e}-01$ & $1.81 \mathrm{e}-06$ & $1.00 \mathrm{e}-05$ \\
BY112 & 0 & $1.00 \mathrm{e}-04$ & $1.94 \mathrm{e}-02$ & $1.49 \mathrm{e}-01$ & $1.94 \mathrm{e}-06$ & $1.49 \mathrm{e}-05$ \\
SX103 & 1 & $1.00 \mathrm{e}-04$ & $1.96 \mathrm{e}-02$ & $1.04 \mathrm{e}-01$ & $1.96 \mathrm{e}-06$ & $1.04 \mathrm{e}-05$ \\
TX115 & 0 & $1.00 \mathrm{e}-04$ & $2.00 \mathrm{e}-02$ & $1.59 \mathrm{e}-01$ & $2.00 \mathrm{e}-06$ & $1.59 \mathrm{e}-05$ \\
BY111 & 0 & $1.00 \mathrm{e}-04$ & $2.11 \mathrm{e}-02$ & $1.56 \mathrm{e}-01$ & $2.11 \mathrm{e}-06$ & $1.56 \mathrm{e}-05$ \\
SX105 & 0 & $1.00 \mathrm{e}-04$ & $2.13 \mathrm{e}-02$ & $1.09 \mathrm{e}-01$ & $2.13 \mathrm{e}-06$ & $1.09 \mathrm{e}-05$ \\
SX104 & 0 & $1.00 \mathrm{e}-04$ & $2.17 \mathrm{e}-02$ & $9.60 \mathrm{e}-02$ & $2.17 \mathrm{e}-06$ & $9.60 \mathrm{e}-06$ \\
BY104 & 4 & $1.00 \mathrm{e}-04$ & $3.01 \mathrm{e}-02$ & $1.30 \mathrm{e}-01$ & $3.01 \mathrm{e}-06$ & $1.30 \mathrm{e}-05$ \\
TX106 & 0 & $1.00 \mathrm{e}-04$ & $3.22 \mathrm{e}-02$ & $1.48 \mathrm{e}-01$ & $3.22 \mathrm{e}-06$ & $1.48 \mathrm{e}-05$ \\
TX102 & 1 & $1.00 \mathrm{e}-04$ & $3.31 \mathrm{e}-02$ & $1.58 \mathrm{e}-01$ & $3.31 \mathrm{e}-06$ & $1.58 \mathrm{e}-05$ \\
S105 & 0 & $1.00 \mathrm{e}-04$ & $3.42 \mathrm{e}-02$ & $1.43 \mathrm{e}-01$ & $3.42 \mathrm{e}-06$ & $1.43 \mathrm{e}-05$ \\
TX105 & 0 & $1.00 \mathrm{e}-04$ & $3.47 \mathrm{e}-02$ & $1.48 \mathrm{e}-01$ & $3.47 \mathrm{e}-06$ & $1.48 \mathrm{e}-05$ \\
\hline
\end{tabular}




\section{Conclusions}

Figure 5 compares the proportions of reactive waste calculated under the three definitions. Each ordinate represents a tank, while the three lines in this figure identify calculations performed using one definition of reactive waste. From this figure one can see that using Region 1 as the definition of reactive waste produces a proportion that is almost always less than one in a hundred. On the other hand, the most conservative definition of reactive waste (Region 2) produces proportions that are in the three out of a hundred range.

When reactive Region 1 is used to define reactive waste, the proportion of reactive waste in most tanks is therefore quite low. For about half the tanks the proportion is less than 1 in a thousand. The "high" tanks. which contain proportions of reactive waste in the range of $1 \%$ are not unreasonable. For example, about $1.5 \%$ of all compiled TOC measurements are above the $4.5 \%$ threshold.

The probabilities associated with reactive Region 1 are most relevant to tank safety. Reactive Region 1 is the criterion used in [3] to define reactive waste. Given this definition, the median fire probabilities are generally below the one-in-a-million threshold normally used to identify unacceptable risk. As one can see, only six tanks have a fire probability higher than one-in-a-million. 


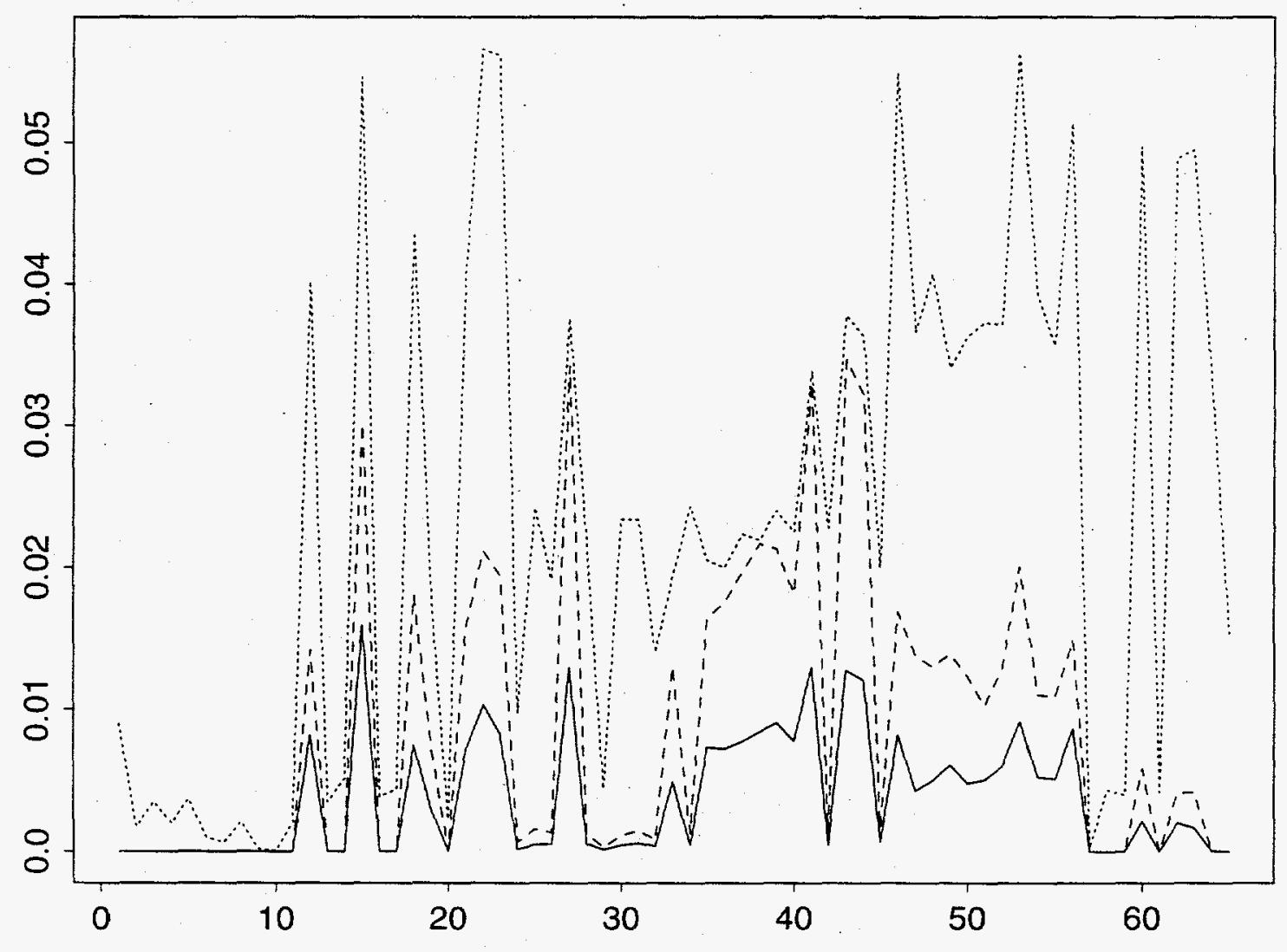

(Region 1=solid, Region 2=dotted, Region 3=dashed)

Figure 2: Proportion of Reactive Waste for Regions 1, 2, and 3. 


\section{References}

[1] Box GEP, Tiao GC, "Bayesian Inference in Statistical Analysis," Addison-Wesley, 1973.

[2] Toth JJ, Heasler PG, Hill JG, Lerchen ME, Whitney PD, "Analysis of Organic Carbon and Moisture in Hanford Single-Shell Tank Waste," PNL-10360, May 1995.

[3] Webb AB, Stewart JL, Turner DA. "Preliminary Safety Criteria for Organic Watch List Tanks at the Hanford Site," WHC-SD-WM-SARR-033, Rev 0, Nov. 95.

[4] "A Safety Assessment for Rotary Mode Core Sampling Operations in Single-Shell Tanks that are on the Flammable Gas Watchlist." Nuclear Systema and Analysis Group. LANL. Draft.

[5] Hanlon, B.M., 1995, Waste Tank Summary Report for Month Ending June 30, 1995, WHC-EP-0182-87, WHC. 


\section{Distribution}

No. of

Copies

\section{OFFSITE}

Los Alamos National Laboratory

P.O. Box 1663

Los Alamos, NM 87545

Attn: W.L. Kubic/K575

\section{ONSITE}

3 DOE Richland Operations Office

J.M. Gray

S7-54

M.F. Jarvis

$\mathrm{K} 8-50$

G.W. Rosenwald
No. of

Copies

18 Pacific Northwest Laboratory

J.W. Brothers

K9-20

P.M. Daling

K8-07

R.T. Hallen

P8-38

P.G. Heasler (3)

K5-12

V.L. Hunter

K7-97

F.M. Ryan

K5-12

J.J. Toth

K7-94

P.D. Whitney

K5-12

M.D. Zentner

K8-37

Information Release

Office (7)

K1-06

8 Westinghouse Hanford Company

H. Babad

L. Jensen

S7-14

N.W. Kirch

J.W. Lentsch

E.J. Lipke

J.E. Meacham

D.A. Reynolds

R.J. Van Vleet
R2-12

R2-11

S7-14

A2-34

S7-14

R2-11

A3-34 
